\title{
JOINT DISTRIBUTION OF THE FIRST HITTING TIME AND FIRST HITTING PLACE FOR A RANDOM WALK
}

\author{
TADASHI NAKAJIMA
}

\begin{abstract}
A random walk on the real line startıng from 0 is considered. A representation of the Lapalace-Fourner transform of the joint distribution of the first hitting time and the first hitting place of the set $(-\infty,-a)(a>0)$ is obtaned, which gives a relation with the joint distribution of those of the set $(-\infty, 0)$. The leading idea is Wiener-Hopf's factorization theorem.
\end{abstract}

\section{Introduction and the main theorem}

Calculation of the distribution of the first hitting time or the first hitting place is closely related to solving the ruin problem in probability theory and boundary value problems for certain difference equations. F. Spitzer [4] studied the distribution of maximum value of a random walk by a combinatorial method. K. Nisioka [3] studied the distribution of the first hitting time and place of a half-line of a biharmonic pseudo process.

In Chapter 18 of [2], W. Feller gave a general formula to calculate the Laplace-Fourier transform of a joint distribution of the first hitting time and the first hitting place of $(-\infty, 0)$ for a general random walk on the real line starting from 0 . We state his result before stating our main theorem.

Let $\left\{X_{n}\right\}$ be independent random variables with a common distribution $F$ and $\left\{S_{n}\right\}$ the random walk generated by $\left\{X_{n}\right\}$. That is, we set

$$
\begin{aligned}
& S_{0}=0, \\
& S_{n}=X_{1}+X_{2}+\cdots+X_{n} .
\end{aligned}
$$

Let $\sigma$ be the first hitting time of $(-\infty, 0)$, that is,

$$
\sigma= \begin{cases}n & \text { if } \min _{j \leq n-1} S_{j} \geq 0 \text { and } S_{n}<0 \\ \infty & \text { otherwise. }\end{cases}
$$

Received November 6, 1997; revised March 5, 1998. 
All sets considered below are Borel sets. We set

$$
H_{n}(A)= \begin{cases}P\left(\sigma=n, S_{\sigma} \in A\right), & A \subset(-\infty, 0) \\ 0, & A \subset[0, \infty) .\end{cases}
$$

For $\lambda \in \boldsymbol{R}$ and $u>0$, we set the Laplace transform of $H_{n}(A)$

$$
H(u, A)=\sum_{n=1}^{\infty} e^{-u n} H_{n}(A)
$$

and the Fourier transform of $H(u, \cdot)$

$$
\chi(u, \lambda)=\int e^{i \lambda x} H(u, d x)
$$

The convolution $F_{1} * F_{2}$ of two finite measures $F_{1}, F_{2}$ on $\boldsymbol{R}$ is defined by

$$
F_{1} * F_{2}(A)=\int F_{1}(A-x) F_{2}(d x) .
$$

The following is known (Feller [2]).

LEMMA 1 ([2], XVIII.3, Lemma 1).

$$
\log \frac{1}{1-\chi(u, \lambda)}=\sum_{n=1}^{\infty} \frac{e^{-u n}}{n} \int_{-\infty}^{0-} e^{i \lambda x} F^{* n}(d x)
$$

Here $F^{* n}$ is the $n$ times convolution of $F$, that is,

$$
F^{* n}(A)=\overbrace{F * \cdots * F}^{n \text { times }}(A) \text {. }
$$

In this paper we denote the Laplace-Fourier transform of a joint distribution of the first hitting time and the first hitting place of $(-\infty,-a)$ by $\chi_{a}(u, \lambda)$. In order to state our result, we prepare some notation.

For a fixed $a>0$, let $\tau$ be the first hitting time of $(-\infty,-a)$, that is,

$$
\tau= \begin{cases}n & \text { if } \min _{j \leq n-1}\left(S_{j}+a\right) \geq 0 \text { and } S_{n}+a<0 \\ \infty & \text { otherwise. }\end{cases}
$$

For $n \in \boldsymbol{N}$ ( $\boldsymbol{N}$ is the set of positive integers), we set

$$
\tilde{H}_{n}(A)= \begin{cases}P\left(\tau=n, S_{\tau} \in A\right), & A \subset(-\infty,-a) \\ 0, & A \subset[-a, \infty) .\end{cases}
$$

We set the Laplace transform

$$
\tilde{H}(u, A)=\sum_{n=1}^{\infty} e^{-u n} \tilde{H}_{n}(A)
$$


and the Fourier transform of $\tilde{H}(u, \cdot)$

$$
\chi_{a}(u, \lambda)=\int e^{i \lambda x} \tilde{H}(u, d x)
$$

Now we state our main theorem.

THeOREM 2. For $u>0$ and $\lambda \in \boldsymbol{R}$,

$$
1-\chi_{a}(u, \lambda)=\frac{1}{2 \pi} \lim _{\varepsilon \downarrow 0} \lim _{N \rightarrow \infty} \int_{-N}^{N} \frac{e^{\imath \varepsilon v}-e^{-i(a+\varepsilon) v}}{i v} \frac{1-\chi(u, \lambda)}{1-\chi(u, \lambda-v)} d v
$$

In what follows, we shall prove this theorem and apply the theorem to an example.

\section{Proof of the main theorem}

We set

$$
\begin{aligned}
& M_{1}=\sigma \\
& M_{l}=\left\{\begin{array}{lll}
n & \text { if } M_{l-1}<n, & \min _{M_{l-1}<j \leq n-1}\left(S_{j}-S_{M_{l-1}}\right) \geq 0 \\
& & \text { and } S_{n}-S_{M_{l-1}}<0
\end{array}\right.
\end{aligned}
$$

where $l=2,3, \ldots$.

For $n \in N$ we set

$$
G_{n}(A)= \begin{cases}0, & A \subset(-\infty, \infty) \backslash[-a, 0) \\ \sum_{j=1}^{n} P\left(M_{J}=n, S_{M_{j}} \in A\right), & A \subset[-a, 0) .\end{cases}
$$

LEMMA 3. For any $A \subset[-a, 0)$, we get

$$
\begin{aligned}
G_{n}(A)= & H_{n}(A)+\sum_{r_{1}+r_{2}=n, r_{1} \geq 1, r_{2} \geq 1} H_{r_{1}} * H_{r_{2}}(A) \cdots \\
& +\sum_{r_{1}+\cdots+r_{k}=n, r_{i} \geq 1} H_{r_{1}} * \cdots * H_{r_{k}}(A)+\cdots
\end{aligned}
$$

Proof. Note that $S_{M_{j-1}} \in[-a, 0)$ if $j \geq 2$ and $S_{M_{j}} \in[-a, 0)$. For $j \geq 2$ and $A \subset[-a, 0)$, we have

$$
\begin{aligned}
P\left(M_{J}\right. & \left.=n, S_{M_{j}} \in A\right) \\
& =\sum_{r=1}^{n-1} P\left(M_{J}=n, S_{M_{j}} \in A, M_{J-1}=r, S_{M_{j-1}} \in[-a, 0)\right) \\
& =\sum_{r=1}^{n-1} \int_{[-a, 0)} P\left(\sigma=n-r, S_{\sigma} \in A-y\right) P\left(M_{J-1}=r, S_{M_{J-1}} \in d y\right) .
\end{aligned}
$$


Therefore we get

$$
\begin{aligned}
G_{n}(A) & =P\left(M_{1}=n, S_{M_{1}} \in A\right)+\sum_{j=2}^{n} P\left(M_{J}=n, S_{M_{j}} \in A\right) \\
& =H_{n}(A)+\sum_{r=1}^{n-1} \int_{[-a, 0)} H_{n-r}(A-y) G_{r}(d y) \\
& =H_{n}(A)+\sum_{r=1}^{n-1} G_{r} * H_{n-r}(A) .
\end{aligned}
$$

Then we get the conclusion by induction.

Lemma 4. For any $A \subset(-\infty,-a)$, we get

$$
\tilde{H}_{n}(A)=H_{n}(A)+\sum_{r+t=n, r \geq 1, t \geq 1} G_{r} * H_{t}(A) .
$$

Proof. We know

$$
\tilde{H}_{n}(A)=H_{n}(A)+\sum_{J=2}^{n} P\left(M_{J}=n, S_{M_{j}} \in A, M_{J-1}<n, S_{M_{j-1}} \geq-a\right) .
$$

Let $j \geq 2$. Since $S_{M_{J-1}}<0$,

$$
\begin{aligned}
P\left(M_{J}\right. & \left.=n, S_{M_{j}} \in A, M_{J-1}<n, S_{M_{j-1}} \geq-a\right) \\
& =\sum_{r=1}^{n-1} P\left(M_{J}=n, S_{M_{j}} \in A, M_{\jmath-1}=r, S_{M_{j-1}} \geq-a\right) \\
& =\sum_{r=1}^{n-1} \int_{[-a, 0)} P\left(\sigma=n-r, S_{\sigma} \in A-y\right) P\left(M_{J-1}=r, S_{M_{j-1}} \in d y\right) .
\end{aligned}
$$

Then we obtain

$$
\tilde{H}_{n}(A)=H_{n}(A)+\sum_{r=1}^{n-1} \int_{[-a, 0)} H_{n-r}(A-y) G_{r}(d y),
$$

which proves the lemma.

For fixed $u>0$, we set

$$
G(u, A)=\sum_{n=1}^{\infty} e^{-u n} G_{n}(A)
$$

Multiplying both sides by $e^{-u n}$ in (4) or (5), and summing up in $n$, we can get the following. 
LeMma 5. For any $A_{1} \subset[-a, 0)$, we get

$$
G\left(u, A_{1}\right)=\sum_{n=1}^{\infty} H^{* n}\left(u, A_{1}\right)
$$

and for any $A_{2} \subset(-\infty,-a)$,

$$
\tilde{H}\left(u, A_{2}\right)=H\left(u, A_{2}\right)+G * H\left(u, A_{2}\right) .
$$

Here $G * H(u, A)=(G * H)(u, A)=(G(u, \cdot) * H(u, \cdot))(A)$ and $H^{* n}(u, A)=\left(H^{* n}\right)$ $(u, A)=H(u, \cdot)^{* n}(A)$.

Now for any $K \subset(-\infty, \infty)$, we set

$$
K_{1}=K \cap[-a, 0)
$$

and

$$
K_{2}=K \cap(-\infty,-a),
$$

and we extend $G(u, A)$ and $\tilde{H}(u, A)$ in the following manner:

$$
G(u, K)=G\left(u, K_{1}\right)
$$

and

$$
\tilde{H}(u, K)=\tilde{H}\left(u, K_{2}\right)
$$

LEMMA 6. For any $K$, we get

$$
\tilde{H}(u, K)+G(u, K)=H(u, K)+G * H(u, K) .
$$

Proof. Let $K_{1}$ and $K_{2}$ be as above. Since $K_{1} \cup K_{2}=K \cap(-\infty, 0)$ and $K_{1} \cap K_{2}=\emptyset$, we see that

$$
\begin{aligned}
& G(u, K)=\sum_{n=1}^{\infty} H^{* n}\left(u, K_{1}\right) \\
& \tilde{H}(u, K)=H\left(u, K_{2}\right)+\sum_{n=1}^{\infty} \int_{[-a, 0)} H^{* n}(u, d y) H\left(u, K_{2}-y\right) .
\end{aligned}
$$

So we obtain

$$
\begin{aligned}
G(u, K)+\tilde{H}(u, K) & =H(u, K)+\sum_{n=1}^{\infty} \int_{[-a, 0)} H^{* n}(u, d y) H(u, k-y) \\
& =H(u, K)+G * H(u, K) .
\end{aligned}
$$

Set

$$
\gamma(u, \lambda)=\int e^{i \lambda x} G(u, d x)+1
$$


Taking Fourier transform of both sides in (6), we get

$$
\chi_{a}(u, \lambda)+\gamma(u, \lambda)=1+\chi(u, \lambda) \gamma(u, \lambda) .
$$

LEMMA 7.

$$
\int_{[-a, 0)} H^{* n}(u, d x) e^{i \lambda x}=\frac{1}{2 \pi} \lim _{\varepsilon \downarrow 0} \lim _{N \rightarrow \infty} \int_{-N}^{N} \frac{e^{\imath \varepsilon v}-e^{-i(a+\varepsilon) v}}{i v} \chi^{n}(u, \lambda-v) d v .
$$

Proof. We have

$$
\begin{aligned}
& \frac{1}{2 \pi} \lim _{\varepsilon \downarrow 0} \lim _{N \rightarrow \infty} \int_{-N}^{N} \frac{e^{i \varepsilon v}-e^{-i(a+\varepsilon) v}}{i v} \chi^{n}(u, \lambda-v) d v \\
& \quad=\frac{1}{2 \pi} \lim _{\varepsilon \downarrow 0} \lim _{N \rightarrow \infty} \int_{-N}^{N} \frac{e^{i \varepsilon v}-e^{-i(a+\varepsilon) v}}{i v} d v \int_{-\infty}^{\infty} H^{* n}(u, d x) e^{i(\lambda-v) x} \\
& \quad=\frac{1}{2 \pi} \lim _{\varepsilon \downarrow 0} \lim _{N \rightarrow \infty} \int_{-\infty}^{\infty} H^{* n}(u, d x) e^{i \lambda x} \int_{-N}^{N} \frac{e^{-i(x-\varepsilon) v}-e^{-i(x+a+\varepsilon) v}}{i v} d v .
\end{aligned}
$$

Applying Dirichlet's integral to this, we get the result.

From Lemmas 5 and 7, we get

\section{LEMMA 8.}

$$
\gamma(u, \lambda)=\frac{1}{2 \pi} \lim _{\varepsilon \downarrow 0} \lim _{N \rightarrow \infty} \int_{-N}^{N} \frac{e^{\ell \varepsilon v}-e^{-i(a+\varepsilon) v}}{i v} \frac{1}{1-\chi(u, \lambda-v)} d v .
$$

Now we show the main theorem in this paper.

Proof of Theorem 2. By (7) we get

$$
1-\chi_{a}(u, \lambda)=(1-\chi(u, \lambda)) \gamma(u, \lambda) \text {. }
$$

Applying this to Lemma 8 , we get the result.

Remark 9. Our conclusion holds for the signed measure $F(d x)$ with total variation $V$ if $V e^{-u}<1$. We will study such a case in [5].

\section{An example}

Let $\left\{X_{l}\right\}$ be independent identically distributed random variables with distribution having the density

$$
\rho(x)= \begin{cases}\frac{\alpha \beta}{\alpha+\beta} e^{\alpha x}, & x<0, \\ \frac{\alpha \beta}{\alpha+\beta} e^{-\beta x}, & x>0,\end{cases}
$$


where $0<\alpha<\beta$. Let $\left\{S_{n}\right\}$ be the random walk generated by $\left\{X_{l}\right\}$. This random walk is frequently appeared in the queuing theory. Customers arrive at rate $\alpha$ and are served at rate $\beta$.

We know

$$
\chi(u, \lambda)=\frac{p(u)}{\alpha+i \lambda}
$$

where $2 p(u)=\alpha+\beta-\sqrt{(\alpha+\beta)^{2}-4 \alpha \beta e^{-u}}$ ([2], XVIII.3, Example c).

We denote

$$
f(v)=\frac{1-\chi(u, \lambda)}{1-\chi(u, \lambda-v)}
$$

and calculate

$$
\begin{aligned}
1-\chi_{a}(u, \lambda) & =\frac{1}{2 \pi} \lim _{\varepsilon \downarrow 0} \lim _{N \rightarrow \infty} \int_{-N}^{N} \frac{e^{i \varepsilon v}-e^{-i(a+\varepsilon) v}}{i v} \frac{1-\chi(u, \lambda)}{1-\chi(u, \lambda-v)} d v \\
& =\frac{1}{2 \pi i} \lim _{\varepsilon \downarrow 0} \lim _{N \rightarrow \infty} \int_{-N}^{N} \frac{e^{i \varepsilon v}-e^{-i(a+\varepsilon) v}}{v} f(v) d v
\end{aligned}
$$

It is easy to see that $p(u)<\alpha$. Therefore $f(v)$ has a pole at $\lambda+i(p(u)-\alpha)$ in the lower half plane.

Let $0<r<R$ and consider the closed curve $C^{1}=C_{1}^{1} \cup C_{2}^{1} \cup C_{3}^{1}$, where

$$
\begin{aligned}
& C_{1}^{1}=\left\{v \in \boldsymbol{C}: v=r e^{i \theta}, 0<\theta<\pi\right\}, \\
& C_{2}^{1}=\{v \in \boldsymbol{R}: R \geq|v| \geq r\}, \\
& C_{3}^{1}=\left\{v \in \boldsymbol{C}: v=\operatorname{Re}^{i \theta}, 0<\theta<\pi\right\} .
\end{aligned}
$$

$C^{1}$ is oriented counterclockwise.

By Cauchy's integral theorem we have

$$
\int_{C^{1}} \frac{e^{\imath \varepsilon v}}{v} f(v) d v=0 .
$$

On the other hand, we have

$$
\begin{aligned}
\left|\int_{C_{3}^{1}} \frac{e^{\imath v}}{v} f(v) d v\right| & =\left|\int_{0}^{\pi} \frac{e^{\imath \varepsilon R e^{i \theta}}}{R e^{i \theta}} f\left(R e^{i \theta}\right) i R e^{i \theta} d \theta\right| \\
& \leq \int_{0}^{\pi} e^{-\varepsilon R \sin \theta}\left|\frac{1-\chi(u, \lambda)}{1-\frac{p(u)}{\alpha+i\left(\lambda-R e^{i \theta}\right)}}\right| d \theta \\
& \rightarrow 0 \quad(\text { as } R \rightarrow \infty) .
\end{aligned}
$$


And we have

$$
\begin{aligned}
\int_{C_{1}^{1}} \frac{e^{l \varepsilon v}}{v} f(v) d v & =\int_{\pi}^{0} \frac{e^{\imath \varepsilon r e^{i \theta}}}{r e^{i \theta}} f\left(r e^{i \theta}\right) i r e^{i \theta} d \theta \\
& =i \int_{\pi}^{0} e^{\imath \varepsilon r e^{i \theta}} \frac{1-\chi(u, \lambda)}{1-\frac{p(u)}{\alpha+i\left(\lambda-r e^{i \theta}\right)}} d \theta \\
& \rightarrow-i \pi \quad(\text { as } r \rightarrow 0) .
\end{aligned}
$$

Thus for a fixed $\varepsilon>0$ we have

$$
\frac{1}{2 \pi i} \lim _{N \rightarrow \infty} \int_{-N}^{N} \frac{e^{\imath \varepsilon v} v}{v} f(v) d v=\frac{1}{2} .
$$

Now we set $C^{2}=C_{1}^{2} \cup C_{2}^{2} \cup C_{3}^{2}$, where

$$
\begin{aligned}
& C_{1}^{2}=\left\{v \in C: v=r e^{i \theta},-\pi<\theta<0\right\}, \\
& C_{2}^{2}=\{v \in \boldsymbol{R}: R \geq|v| \geq r\}, \\
& C_{3}^{2}=\left\{v \in \boldsymbol{C}: v=R e^{i \theta},-\pi<\theta<0\right\} .
\end{aligned}
$$

$C^{2}$ is also oriented counterclockwise.

Since $v=\lambda-i \alpha+i p(u)$ is a pole of order 1 of $f(v)$, by the residue theorem we have

$$
\int_{C^{2}} \frac{e^{-i(a+\varepsilon) v}}{v} f(v) d v=-2 \pi i \frac{p(u)}{\alpha+i \lambda} e^{-i(a+\varepsilon)(\lambda+i(p(u)-\alpha))} .
$$

On the other hand

$$
\begin{aligned}
\left|\int_{C_{3}^{2}} \frac{e^{-i(a+\varepsilon) v}}{v} f(v) d v\right| & =\left|\int_{-\pi}^{0} \frac{e^{-i(a+\varepsilon) R e^{i \theta}}}{R e^{i \theta}} f\left(R e^{i \theta}\right) i R e^{i \theta} d \theta\right| \\
& \leq \int_{-\pi}^{0} e^{(a+\varepsilon) R \sin \theta}\left|\frac{1-\chi(u, \lambda)}{1-\frac{p(u)}{\alpha+i\left(\lambda-\operatorname{Re}^{i \theta}\right)}}\right| d \theta \\
& \rightarrow 0 \quad(\text { as } R \rightarrow \infty) .
\end{aligned}
$$

And for a fixed $\varepsilon>0$

$$
\begin{aligned}
\int_{C_{1}^{2}} \frac{e^{-i(a+\varepsilon) v}}{v} f(v) d v & =\int_{0}^{-\pi} \frac{e^{-i(a+\varepsilon) r e^{i \theta}}}{r e^{i \theta}} f\left(r e^{i \theta}\right) i r e^{i \theta} d \theta \\
& =i \int_{0}^{-\pi} e^{-i(a+\varepsilon) r e^{i \theta}} \frac{1-\chi(u, \lambda)}{1-\frac{p(u)}{\alpha+i\left(\lambda-r e^{i \theta}\right)}} d \theta \\
& \rightarrow-i \pi \quad(\text { as } r \rightarrow 0) .
\end{aligned}
$$


Then we have

$$
\frac{1}{2 \pi i} \lim _{N \rightarrow \infty} \int_{-N}^{N} \frac{e^{-i(a+\varepsilon) v}}{v} f(v) d v=-\frac{1}{2}+\frac{p(u)}{\alpha+i \lambda} e^{-i(a+\varepsilon)(\lambda+i(p(u)-\alpha))} .
$$

Thus we get

$$
\chi_{a}(u, \lambda)=\frac{p(u)}{\alpha+i \lambda} e^{-l a(\lambda+i(p(u)-\alpha))}
$$

This formula is the Laplace-Fourier transform of the distribution of $\tau$ and $S_{\tau}$. In the queing theory, the $\tau$-th customer is the first customer finding out the server who has kept free for time interval of length $-S_{\tau}$ which is longer than $a$.

Acknowledgment. I am very grateful to Professor M. Motoo and Professor S. Sato for their valuable discussion and guidance.

\section{REFERENCES}

[1] FelLeR, W., An Introduction to Probability Theory and Its Applications I, 3-rd edition, John Wiley \& Sons, inc, 1968.

[2] FelleR, W., An Introduction to Probability Theory and Its Applications II, 2-nd edition, John Wiley \& Sons, inc, 1971.

[3] NishoKa, K., The first hitting time and place of a half-line by a biharmonic pseudo process, Japan. J. Math., 23 (1958), 235-280.

[4] SPITZER, F., A combinatorial lemma and its application to probability theory, Trans. Amer. Math. Soc., 82 (1958), 323-339.

[5] Sato, S. and Nakajma, T., On the joint distribution of the first hitting time and the first hitting place to the space-time wedge domain of a biharmonic pseudo process, to appear in Tokyo J. Math., 22 (1999).

DePARTMENT OF INFORMATION SCIENCES

College of SCIENCE AND ENGINEERING

TOKYO DENKI UNIVERSITY

SAITAMA 350-0394

JAPAN 